\title{
Immunoglobulin G4-Hepatopathy with Acute Hepatitis-Like Onset and Marked Centrilobular Necrosis: Clinicopathologically Unique Pattern of Hepatic Injury Related to Immunoglobulin G4-Related Disease
}

\author{
Kaori Mukai ${ }^{a}$ Tsutomu Nishida ${ }^{a}$ Shiro Adachi ${ }^{b}$ Kengo Matsumoto ${ }^{a}$ \\ Naoto Osugi ${ }^{a} \quad$ Aya Sugimoto $^{a}$ Dai Nakamatsu $^{a}$ Masashi Yamamoto $^{a}$ \\ Koji Fukui ${ }^{a}$ Hiromi Tamurab $^{b}$ Masami Inada ${ }^{a}$ \\ aDepartment of Gastroenterology and Hepatology, Toyonaka Municipal Hospital, Toyonaka, \\ Japan; bepartment of Pathology, Toyonaka Municipal Hospital, Toyonaka, Japan
}

\section{Keywords}

Acute hepatitis · Autoimmune hepatitis · Immunoglobulin G4-hepatopathy · Azathioprine

\begin{abstract}
A 69-year-old man presented with jaundice and appetite loss. Blood analyses showed elevated aminotransferase levels, hyperbilirubinemia, positivity for antinuclear antibody, elevated immunoglobulin (lg) G4 levels, and negativity for hepatitis virus markers. Additionally, computed tomography revealed a focal enlargement of the pancreatic body and enhancement of the peripheral bile ducts. Liver biopsy showed interface hepatitis, supporting a clinical diagnosis of autoimmune hepatitis (AIH). Immunohistochemistry revealed that IgG4-bearing plasma cells accounted for more than $60 \%$ of the lgG-bearing plasma cells in the portal area. Then, we started oral prednisolone therapy. After tapering, serum transaminase levels became elevated again, and we had to adjust the dose. Azathioprine maintenance therapy was necessary to prevent relapse. We herein report a case of IgG4-hepatopathy with a clinical course similar to that of AlH with acute onset.
\end{abstract}




\section{Case Reports in Gastroenterology}

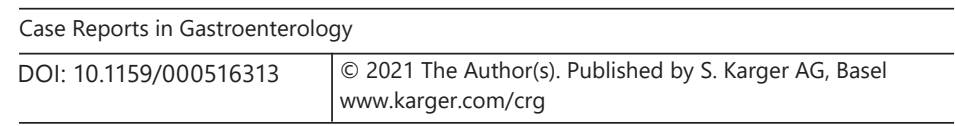

Mukai et al.: Immunoglobulin G4-Hepatopathy with Acute Hepatitis-Like Onset

\section{Introduction}

Immunoglobulin (Ig) G4-related disease (RD) is now a widely acknowledged systemic disease involving many organs, especially the pancreatobiliary and salivary systems [1]. Although clinical manifestations may vary according to the involved organs, the essential histopathological findings are relatively consistent, regardless of the affected organs [2]. Therefore, the diagnosis is usually made based on the findings of histopathological examination, except for the typical cases of IgG4-related autoimmune pancreatitis (AIP). In the hepatobiliary system, IgG4 sclerosing cholangitis (SC) mainly involving extrahepatic common bile duct and IgG4-related hepatic pseudotumor in the hilar region are considered parts of the spectrum of IgG4-RD [2]. Type-1 AIP is a prototypic manifestation of IgG4-RD [3]. The affected pancreas shows diffuse or nodular enlargement and narrowing of the main pancreatic duct histopathologically characterized by IgG4-bearing plasma cell infiltration, storiform fibrosis, and obliterative phlebitis. Liver dysfunction has been known in patients with type-1 AIP [4]. Umemura et al. [5] demonstrated that classic autoimmune hepatitis (AIH)-like lesions with IgG4-bearing plasma cells were found in the livers of the patients with type-1 AIP called "IgG4-hepatopathy." Here, we report a unique case of a patient with type-1 AIP whose clinical manifestation was an acute onset of severe hepatitis and whose histopathological findings were consistent with IgG4-hepatopathy with an unprecedented magnitude of centrilobular collapse. Glucocorticoids were the first-line therapy, and azathioprine (AZA) was a useful second therapy.

\section{Case Report}

A 64-year-old man presented at our hospital with appetite loss, jaundice, and brown urine. Laboratory data showed elevated transaminase and bilirubin levels. He was diagnosed with acute hepatitis. The laboratory data at admission were as follows: aspartate aminotransferase, 1,592 U/L [reference range: 10-37]; alanine aminotransferase (ALT), 1,475 U/L [4-40]; alkaline phosphatase 1,375 U/L [98-328]; g-glutamyl transpeptidase, 522 U/L [11-64]; total bilirubin, $14.0 \mathrm{mg} / \mathrm{dL}$ [0.2-1.2]; direct bilirubin, $11 \mathrm{mg} / \mathrm{dL}$ [0-0.3]; PT, 66\% [70-130]; and pancreatic amylase, $138 \mathrm{U} / \mathrm{L}$ [14-41]. His serum IgG level was 3,452 mg/dL [870-1,700], and his IgG4 level was $1,250 \mathrm{mg} / \mathrm{dL}$ [4.8-105]. Antinuclear antibody was positive, with a slightly high titer at 1:40 and a nucleolar pattern, and the antimitochondrial antibody was negative (Table 1). The patient reported no habit of drinking alcohol and did not take any drugs or herbal remedies. Infection with hepatitis B and C was excluded. There was no specific past medical history, except for chronic atrophic gastritis and prostatic hyperplasia as comorbid diseases. Contrasted enhanced computed tomography showed enhancement of the peripheral bile ducts in the left lobe of the liver and thickening of the wall of the common bile duct (Fig. 1a). Bile duct obstruction was not observed. Moreover, contrasted enhanced computed tomography revealed that the pancreas had a capsule-like rim and showed decreased enhancement in the body in the pancreatic phase and narrowing of the main pancreatic duct (Fig. 1b). T2-weighted magnetic resonance imaging showed narrowing of the main pancreatic duct in the body and swelling of the pancreas, but not irregularity of bile ducts (Fig. 1c, d). We performed a liver biopsy, and the histological examination revealed extensive collapse of the centrilobular parenchyma in addition to scattered acidophilic bodies in the lobular area, moderate interface hepatitis, and hepatocyte rosetting in the portal area (Fig. 2a). In the portal area, there was moderate lymphoplasmacytic infiltrate with plasma cells predominant and mild eosinophilic infiltrate (Fig. 2b). In the collapsed centrilobular area, a similar composition of the inflammatory cells was found, though the degree of the infiltration was much less

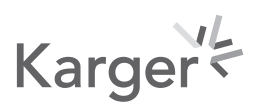


Case Reports in Gastroenterology

Table 1. Laboratory data at admission

\section{Biochemical data}

$\mathrm{WBC}, / \mu \mathrm{L}$

6,800

$\mathrm{RBC}, \times 10^{4} / \mu \mathrm{L}$

439

$\mathrm{Hb}, \mathrm{g} / \mathrm{dL}$

Platelets, $\times 10^{4} / \mu \mathrm{L}$

10.0

PT, \% 66

PT-INR

D-dimer, $\mu \mathrm{g} / \mathrm{mL}$

AST, U/L

ALT, U/L

1,475

LDH, U/L

500

ALP, U/L

1,375

g-GTP, U/L

522

Alb, g/dL

T-bil, mg/dL

14.0

D-bil, mg/dL

BUN, mg/dL

AMY, U/L

P-AMY, U/L 138

$\mathrm{Cr}, \mathrm{mg} / \mathrm{dL}$

$\mathrm{UA}, \mathrm{mg} / \mathrm{dL}$

CRP, mg/dL

$\mathrm{Na}, \mathrm{mEq} / \mathrm{L}$

$\mathrm{K}, \mathrm{mEq} / \mathrm{L}$

Immunological and other data

$\mathrm{IgG}, \mathrm{mg} / \mathrm{dL}$

3,452

IgM, mg/dL

67

$\mathrm{IgA}, \mathrm{mg} / \mathrm{dL}$

241

IgG4, mg/dL

1,250

ANA

40

AMA

TSH, $\mu \mathrm{IU} / \mathrm{mL}$

Free T4, ng/dL

Free T3, pg/mL

Viral markers

HBsAg, IU/mL

$\mathrm{HBcAb}, \mathrm{S} / \mathrm{CO}$

$\mathrm{HBsAb}, \mathrm{mIU} / \mathrm{mL}$

HCVAb, S/CO

HAVIgM, S/CO $<0.40$

ALT, alanine aminotransferase; PT, prothrombin time; T-bil, total bilirubin; Ig, immunoglobulin; AST, aspartate aminotransferase; ALP, alkaline phosphatase; g-GTP, g-glutamyl transpeptidase; ANA, antinuclear antibody; AMA, antimitochondrial antibody. 

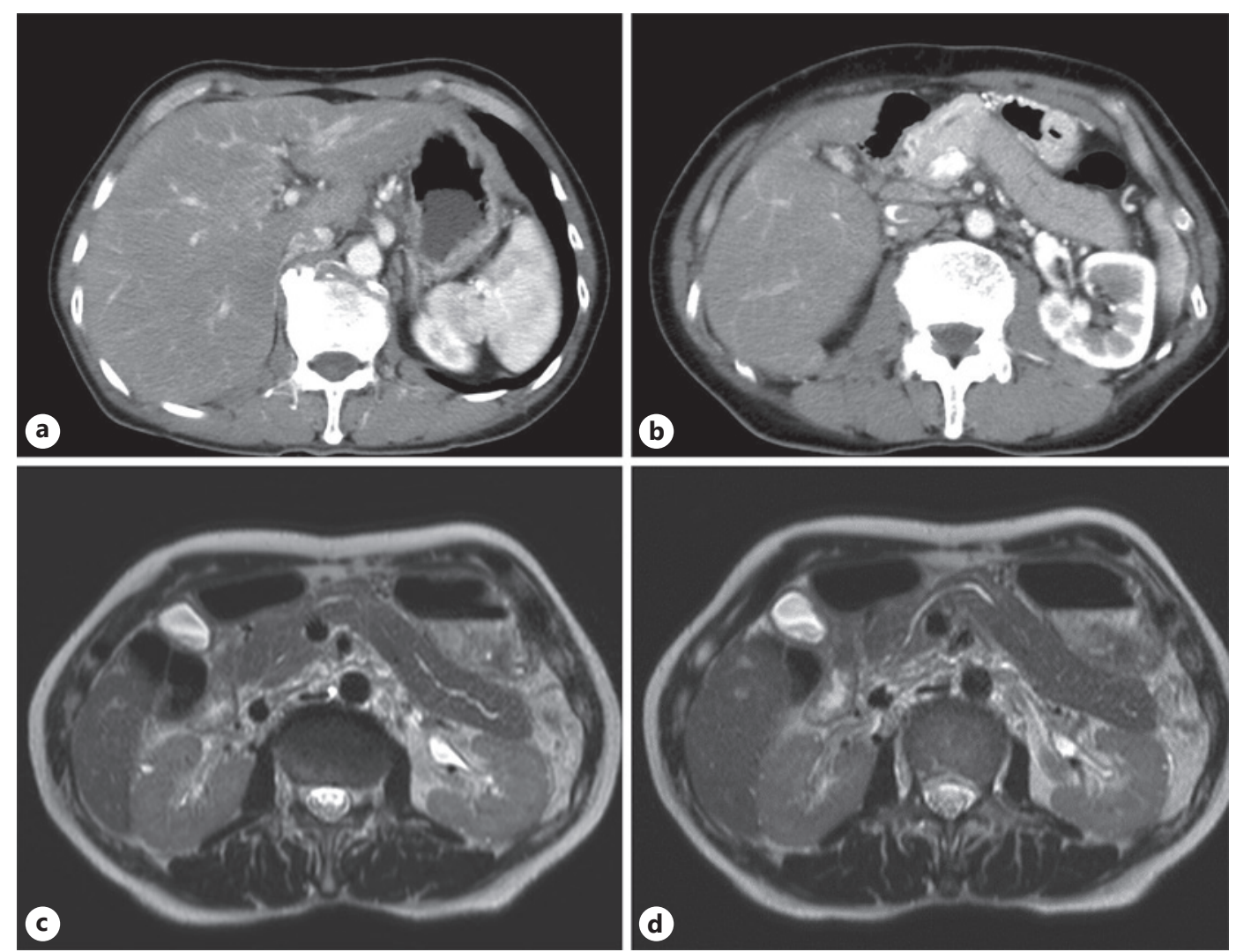

Fig. 1. Image findings on CE-CT and MRI. a Enhancement of the peripheral bile ducts in the left lobe of the liver and thickening of the wall of the common bile duct. $\mathbf{b}$ Decrease of enhancement of the pancreas in the body and the narrowing of the main pancreatic duct. $\mathbf{c}, \mathbf{d}$ Narrowing of the main pancreatic duct and swelling of the pancreas. CE-CT, contrasted enhanced computed tomography; MRI, magnetic resonance imaging.

than that in the portal area. Damage to the interlobular bile ducts and cholestasis was not found (Fig. 2c). Although bridging necrosis and fibrosis were observed, storiform fibrosis and obliterative phlebitis were not evident. The laboratory and imaging tests confirmed the diagnosis of type-1 AIP based on the international consensus diagnostic criteria for AIP (guidelines of the International Association of Pancreatology). Moreover, classic AIH was suspected based on the diagnostic criterion provided by the International Autoimmune Hepatitis Group (IAIHG).

In order to reveal the relationship between AIP and acute hepatitis, we performed further histopathological examination. Immunohistochemical analysis revealed that almost all of the plasma cells infiltrating in the portal tract produced IgG; IgG4-bearing plasma cell infiltration was 40-60 cells/HPF per portal area, and the IgG4+/IgG+ ratio was more than $60 \%$ (Fig. $2 \mathrm{~d}$ ). Therefore, hepatic injury was considered as IgG4-hepatopathy.

We started treatment with prednisolone (PSL) at an initial dose of $50 \mathrm{mg} / \mathrm{day}$, and the levels of aminotransferase, IgG, and IgG4 decreased to the normal range for 3 months. Maintenance therapy with PSL of $5 \mathrm{mg}$ /day kept his liver function tests normal (Fig. 3a). He demonstrated relapse 8 months after starting PSL therapy, and his serum ALT was re-elevated along with elevation of IgG4 (113 mg/dL). We increased the PSL dose from 5 to $15 \mathrm{mg}$, and his serum ALT was rapidly normalized; therefore, we were able to taper the dose. However, after tapering, his serum ALT re-elevated, and we had to adjust the dose. After the fourth elevation, we considered him to have become steroid-dependent. Therefore, we added AZA as an immu- 


\section{Case Reports in Gastroenterology}

\begin{tabular}{l|l}
\hline Case Reports in Gastroenterology \\
\hline DOI: 10.1159/000516313 & $\begin{array}{l}\text { @ } 2021 \text { The Author(s). Published by S. Karger AG, Basel } \\
\text { www.karger.com/crg }\end{array}$ \\
\hline
\end{tabular}

Mukai et al.: Immunoglobulin G4-Hepatopathy with Acute Hepatitis-Like Onset
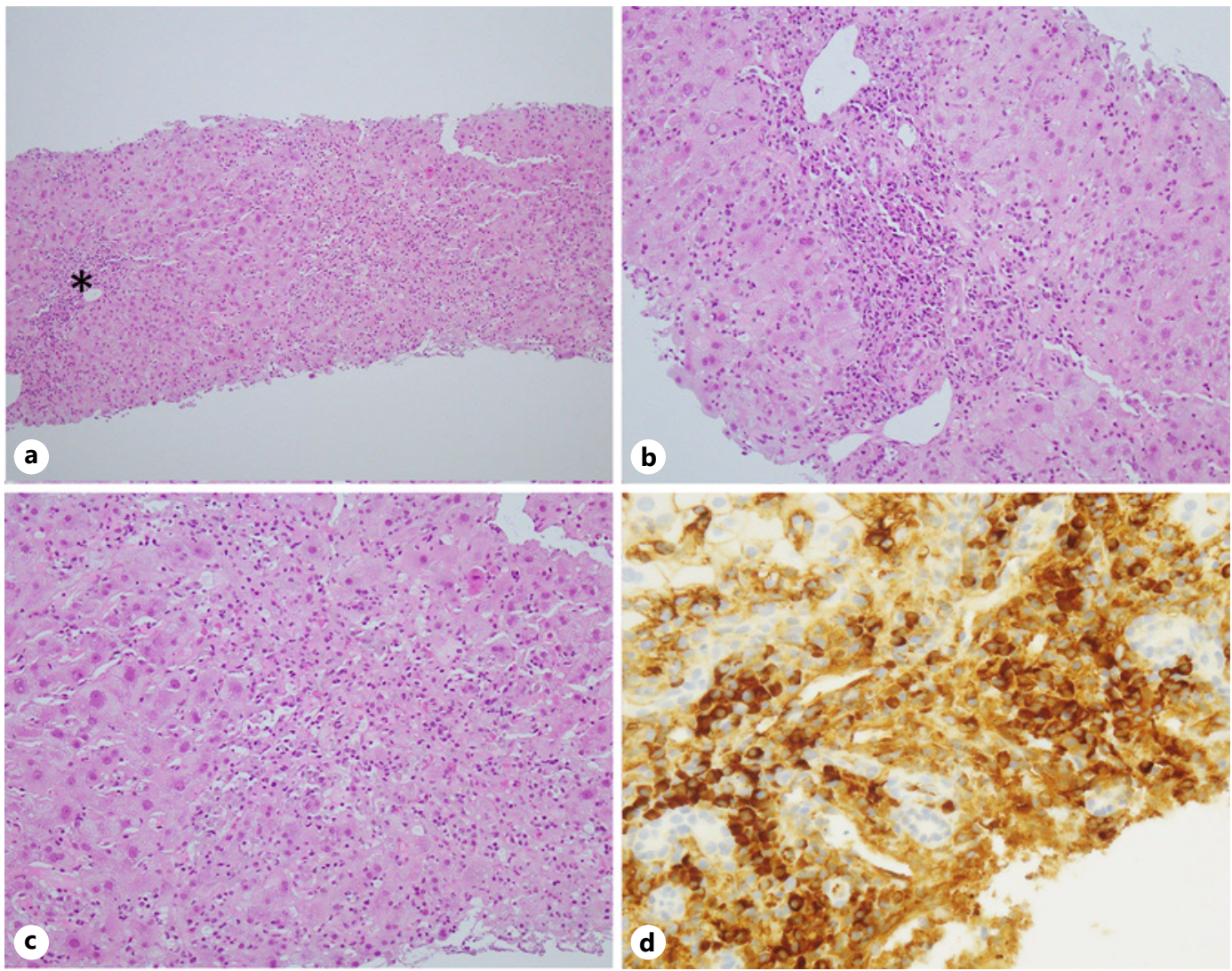

Fig. 2. Histopathological findings of the liver. a Massive centrilobular collapse and moderate portal inflammatory change. Asterisk indicates the portal region (H\&E stain. $\times 100)$. $\mathbf{b}$ In the portal region, interface hepatitis with lymphoplasmacytic infiltrate is observed (H\&E stain. $\times 200$ ). c In the centrilobular region, marked zonal necrosis and scattered acidophilic bodies are observed (H\&E stain. $\times 400$ ). $\mathbf{d}$ Immunohistochemical examination revealed that most plasma cells expressed IgG4, and the ratio of IgG4+/IgG plasma cells was more than $60 \%$ (IgG4 stain. $\times 400$ ). Ig, immunoglobulin.

nomodulator with increased PSL. A $50 \mathrm{mg}$ dose of AZA caused nausea, and the dose was reduced to $25 \mathrm{mg}$. During a relapse of hepatitis, the IgG and IgG4 levels were not elevated. However, steroid therapy decreased his liver dysfunction. After increasing the dose of PSL, his serum ALT was re-normalized, and we were able to taper the dose again (Fig. 3b).

\section{Discussion}

We report a unique case of IgG4-hepatopathy closely resembling classic AIH with marked centrilobular necrosis. It should be specifically mentioned that the presented case manifested clinically as acute hepatitis-like onset, and histopathologically showed the massive collapse of centrilobular hepatocytes, which appeared to correspond to the markedly elevated serum concentration of aminotransferases, as well as chronic active hepatitis with classical features of AIH. Although there have been several studies of IgG4-hepatopathy, no cases showing elevated serum levels of aminotransferase over 1,000 U/L and massive lobular necrosis equivalent to the present case have been reported.

The biliary tract is the most commonly involved extrapancreatic site of IgG4-RD in the patients with type- 1 AIP. Approximately $60-80 \%$ of patients with type- 1 AIP have biliary 


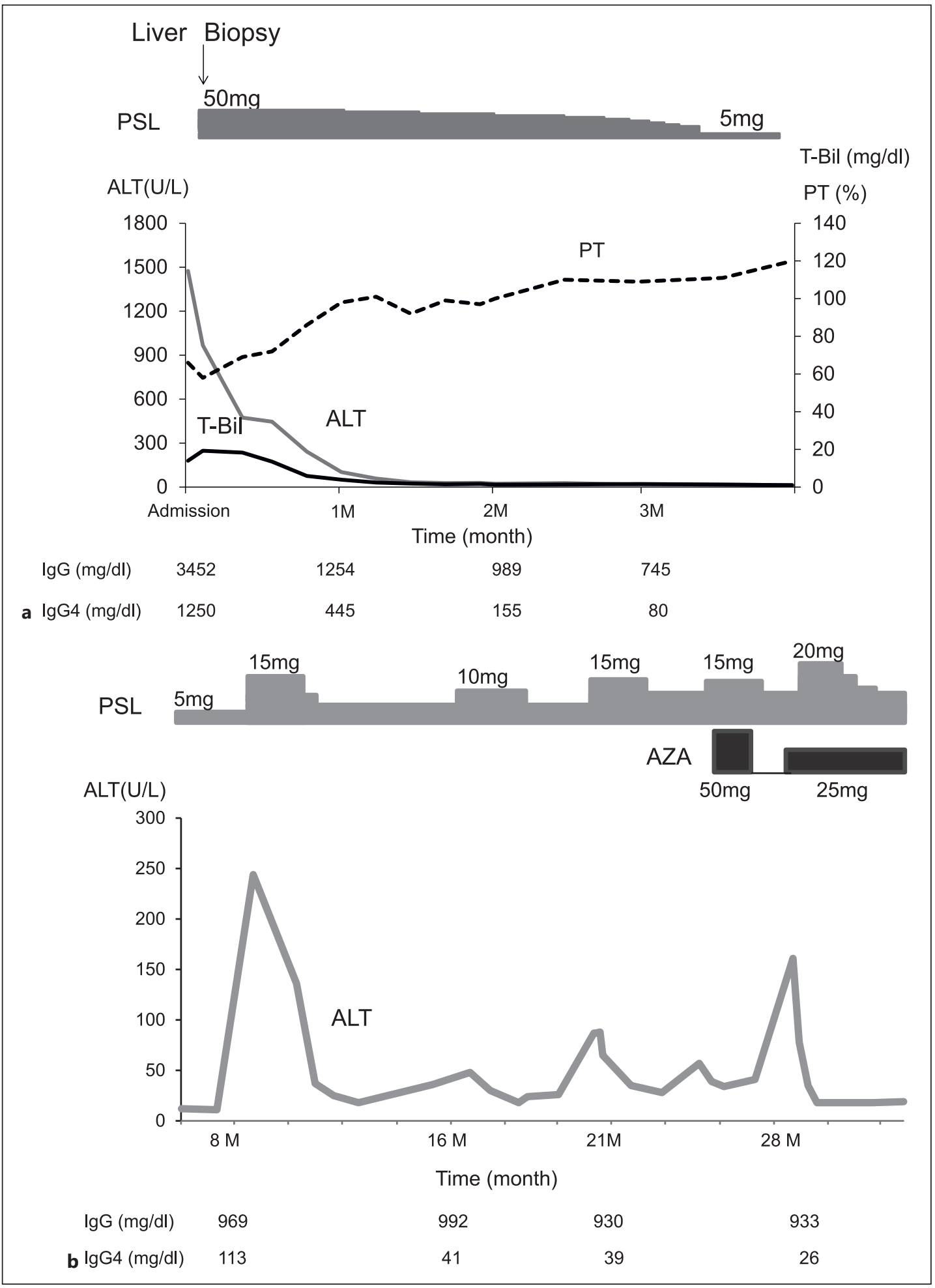

Fig. 3. a Clinical course of changes in serum ALT, PT, T-bil, and IgG4/IgG. Gray boxes indicate PSL treatment. b Clinical course of changes in serum ALT and IgG4/IgG. Gray boxes indicate PSL treatment, and black boxes correspond to AZA administration. ALT, alanine aminotransferase; PT, prothrombin time; T-bil, total bilirubin: Ig, immunoglobulin; PSL, prednisolone; AZA, azathioprine. 
tract involvement, now known as IgG4-SC [6]. Whereas liver dysfunction with elevated liver enzymes is commonly observed in patients with type-1 AIP and/or IgG4-SC [4, 7], the hepatic involvement of these autoimmune diseases remains poorly understood, and there is still confusion in its terminology. Three patterns of IgG4-related hepatic lesion have been described. One is the intrahepatic small bile duct lesion, probably corresponding to the intrahepatic counterpart of IgG4-SC [6]. The second type of hepatic manifestation is hepatic inflammatory pseudotumor [8]. It manifests as a periductal mass, typically involving hilar/perihilar ducts. The last is the pattern of chronic active hepatitis that includes primary or secondary changes related to type-1 AIP and/or IgG4-SC [4, 5, 7, 9]. Umemura et al. [4] performed a clinicopathologic analysis of hepatic manifestation in the patients with type-1 AIP and revealed that a significant proportion of patients exhibited clinicopathologic features closely resembling those of classic $\mathrm{AIH}$, and their liver tissues were infiltrated by a significant number of IgG4-bearing plasma cells [4]. They called these lesions "IgG4-hepatopathy." Harada and Nakanuma [10] also examined the histopathological changes in the liver of patients with IgG4-SC and found chronic hepatitis patterns consistent with classic AIH. Although discrimination between the primary or secondary lesion to IgG4-SC and/or type-1 AIP is difficult, the histopathological findings can be explained as secondary changes by the biliary involvement of IgG4-SC and/or type-1 AIP. Therefore, they may not be specifically related to the histogenesis of IgG4-RD. On the other hand, there have been only 3 cases of "IgG4-associated AIH," which Umemura et al. $[4,7]$ identified when no other IgG4-RD including type-1 AIP was found at the hepatic disease onset [9]. These cases are characterized by elevated serum IgG4 levels and abundant IgG4-bearing plasma cells (IgG4+ cells $>10 / \mathrm{HPF}$ and IgG4+/IgG > 40\%). Interestingly, 2 of them subsequently developed IgG4-RD [4, 9]. The cases so far reported as IgG4hepatopathy or IgG4-associated AIH exhibited a chronic active hepatitis pattern but not parenchymal necroinflammation changes such as zonal, bridging, and broad collapse. It is difficult to definitely classify the present case since it could be caused either by a primary hepatic lesion in a spectrum of IgG4-RD or by a secondary lesion following a change in type-1 AIP. Either way, our case should be categorized as IgG4-hepatopathy.

Chung et al. [11] examined the presence of IgG4-bearing plasma cells in the liver of classic AIH patients and found $35 \%$ of the cases had $>5$ IgG4-bearing plasma cells/HPF. Yeoman et al. [12] also demonstrated that the liver tissue of $11 \%$ of AIH had $>10$ IgG4-bearing plasma cells / HPF. In both studies, however, the proportion of IgG4+ plasma cells did not exceed $40 \%$, and serum IgG4 concentration was not elevated. Moreover, no patients in the studies were associated with other IgG4-RD. Therefore, these cases appear to be a variant of AIH, rather than "IgG4-associated AIH." In order to avoid confusion, we think that the term "IgG4-associated AIH" should be restricted to cases fulfilling the criteria proposed by Umemura et al. [13]: (1) histologically proven active hepatitis, (2) IgG4-positive plasma cell infiltration ( $>10$ cells/hpf; >40\% IgG4/IgG-positive plasma cells), (3) serum elevated IgG4 levels, and (4) lack of SC and AIP.

Whereas IgG4-hepatopathy and IgG4-associated AIH are hepatic lesions with a close clinicopathologic resemblance to AIH $[4,5,7,9]$, there have been no cases showing the pattern of chronic interface hepatitis with marked centrilobular necrosis seen in the present case. Although the fundamental nature of IgG4-hepatopathy and IgG4-associated AIH is not clarified, treatment with steroid therapy ameliorates both hepatic disorders as well as type-1 AIP/IgG4-SC. Glucocorticoids are preferred as a first-line therapy (mostly prednisone 0.5-1 $\mathrm{mg} / \mathrm{kg} /$ day); however, the relapse rate after tapering glucocorticoid is high [14]. Therefore, steroid-sparing maintenance therapy is often required. There are no formal treatment guidelines; however, disease-modifying antirheumatic drugs such as AZA, methotrexate, mycophenolate mofetil, hydroxychloroquine, and cyclophosphamide were considered as second-line treatments. AZA should be considered as a second-line treatment in cases affecting the liver

\section{Karger's}


and pancreas [15]. Further cases need to be studied to evaluate the relationship between IgG4-RD and hepatic disorders and steroid-sparing treatment.

\section{Acknowledgements}

The authors thank Dr. Yoh Zen (Institute of Liver Studies, Kings College Hospital of London, UK) for discussing the case and encouraging us to record it.

\section{Statement of Ethics}

All procedures were performed in accordance with the ethical standards of the responsible committee on human experimentation (institutional and national) and with the Helsinki Declaration of 1975, as revised in 2008. Written informed consent was obtained from the patient for publication of this case report and any accompanying images. It is the policy of the Institutional Review Board (IRB) of the Toyonaka Municipal Hospital that a "single case report" or "limited case series" does not require review by the IRB. The IRB approval was waived for this case report.

\section{Conflict of Interest Statement}

The authors have no conflicts of interest to declare.

\section{Funding Sources}

This case report has no funding sources.

\section{Author Contributions}

K. Mukai wrote the manuscript. K. Mukai, K. Matsumoto, K. Fukui, S. Adachi, and H. Tamura diagnosed and treated the patient. S. Adachi and T. Nishida helped to draft the manuscript and revised it critically. All authors read and approved the final version of the manuscript.

\section{References}

1 Stone JH, Zen Y, Deshpande V. IgG4-related disease. N Engl J Med. 2012;366(6):539-51.

2 Deshpande V, Zen Y, Chan JK, Yi EE, Sato Y, Yoshino T, et al. Consensus statement on the pathology of IgG4related disease. Mod Pathol. 2012;25(9):1181-92.

3 Kamisawa T, Chari ST, Lerch MM, Kim MH, Gress TM, Shimosegawa T. Recent advances in autoimmune pancreatitis: type 1 and type 2. Gut. 2013;62(9):1373-80.

4 Umemura T, Zen Y, Nakanuma Y, Kiyosawa K. Another cause of autoimmune hepatitis. Hepatology. 2010; 52(1):389-90.

5 Umemura T, Zen Y, Hamano H, Kawa S, Nakanuma Y, Kiyosawa K. Immunoglobin G4-hepatopathy: association of immunoglobin G4-bearing plasma cells in liver with autoimmune pancreatitis. Hepatology. 2007;46(2): 463-71.

6 Zen Y, Nakanuma Y, Portmann B. Immunoglobulin G4-related sclerosing cholangitis: pathologic features and histologic mimics. Semin Diagn Pathol. 2012;29(4):205-11.

\section{Karger's}


7 Umemura T, Zen Y, Hamano H, Ichijo T, Kawa S, Nakanuma Y, et al. IgG4 associated autoimmune hepatitis: a differential diagnosis for classical autoimmune hepatitis. Gut. 2007;56(10):1471-2.

8 Zen Y, Harada K, Sasaki M, Sato Y, Tsuneyama K, Haratake J, et al. IgG4-related sclerosing cholangitis with and without hepatic inflammatory pseudotumor, and sclerosing pancreatitis-associated sclerosing cholangitis: do they belong to a spectrum of sclerosing pancreatitis? Am J Surg Pathol. 2004;28(9):1193-203.

9 Ishizu Y, Ishigami M, Kuzuya T, Honda T, Hayashi K, Nakano I, et al. Immunoglobulin G4-associated autoimmune hepatitis later complicated by autoimmune pancreatitis: a case report. Hepatol Res. 2016;46(6):601-6.

10 Harada K, Nakanuma Y. Pathological differences between IgG4-related sclerosing cholangitis with and without autoimmune pancreatitis. Mod Pathol. 2013;25(2):403A.

11 Chung H, Watanabe T, Kudo M, Maenishi O, Wakatsuki Y, Chiba T. Identification and characterization of IgG4associated autoimmune hepatitis. Liver Int. 2010;30(2):222-31.

12 Yeoman AD, Zen Y, Heneghan MA. PTU-017 IgG4 +ve autoimmune hepatitis is not observed among patients of non-Asian origin. Gut. 2012;61(Suppl 2):1.

13 Umemura T, Zen Y, Hamano H, Joshita S, Ichijo T, Yoshizawa K, et al. Clinical significance of immunoglobulin G4-associated autoimmune hepatitis. J Gastroenterol. 2011;46(Suppl 1):48-55.

14 Kamisawa T, Okazaki K, Kawa S, Ito T, Inui K, Irie H, et al. Amendment of the Japanese consensus guidelines for autoimmune pancreatitis, 2013. III. Treatment and prognosis of autoimmune pancreatits. J Gastroenterol. 2014;49:961-70.

15 Khosroshahi A, Wallace ZS, Crowe JL, Akamizu T, Azumi A, Carruthers MN, et al. International consensus guidance statement on the management and treatment of IgG4-related disease. Arthritis Rheumatol. 2015; 67(7):1688-99. 